\title{
Pontryagin trace anomaly
}

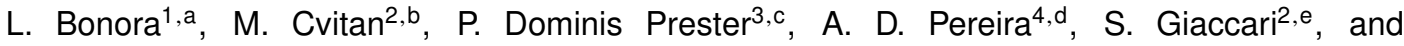 \\ T. Štemberga ${ }^{2, f}$ \\ ${ }^{1}$ International School for Advanced Studies (SISSA), Via Bonomea 265, 34136 Trieste, Italy, and INFN, \\ Sezione di Trieste \\ ${ }^{2}$ Department of Physics, Faculty of Science, University of Zagreb, Bijenička cesta 32, 10000 Zagreb, Croatia \\ ${ }^{3}$ Department of Physics, University of Rijeka, Radmile Matejčić 2, 51000 Rijeka, Croatia \\ ${ }^{4}$ Institut für Theoretische Physik, Universität Heidelberg, Philosophenweg 12, 69120 Heidelberg, Germany
}

\begin{abstract}
We review the recent results on the Pontryagin trace anomaly in QFT's with free Weyl fermion in curved four-dimensional spacetime.
\end{abstract}

\section{Introduction: quantum anomalies}

After almost 90 years of development we are still not satisfied with our understanding of quantum field theory. As (quantum) anomalies are a genuine quantum property, studying them is essential in our quest for the answer to the question "What is QFT?". And, as we shall argue here for the case of trace anomalies, one may find big surprises in such studies.

Anomalies can be divided into two types, harmless and harmful ones. Harmless anomalies may produce interesting effects, such as violations of classical (global) symmetries, with important consequences, both theoretical and phenomenological, but they leave QFT's well defined and consistent. On the other hand, harmful anomalies, such as those violating standard gauge symmetries, (apparently) destroy consistency of QFT. In gauge and gravity theories harmful anomalies are identified with consistent and split anomalies, i.e. anomalies that satisfy the Wess-Zumino consistency conditions and have opposite sign for opposite chiralities. Covariant gauge anomalies, as the name says, behave covariantly under the relevant symmetry and are non-split, i.e. they have the same sign for opposite chiralities and are harmless. Even parity trace anomalies (see below) are consistent under Weyl transformation and diffeomorphism covariant, they are non-split and harmless. What we point out here is that there may exist an odd parity trace anomaly, which is consistent under Weyl transformation and diffeomorphism covariant, but is split and potentially harmful.

In calculations of anomalies we are guided and helped by the following properties they usually satisfy: (i) consistency conditions, leaving a small number of potential candidates for the anomaly,

\footnotetext{
a e-mail: bonora@sissa.it

be-mail: mcvitan@phy.hr

ce-mail: pprester@uniri.hr

de-mail: a.pereira@thphys.uni-heidelberg.de

e-mail: sgiaccari@phy.hr

fe-mail: tstember@phy.hr
} 
(ii) one-loop completeness. However, one should be careful here. Though the property (ii) is valid for the anomalies in gauge field theories, which are the most frequently studied type of anomalies, it is not generally true. For example, it is well established that the trace anomaly, which is the subject of this paper, is not necessarily 1-loop saturated.

\section{From the Weyl symmetry to the trace anomaly}

The trace anomaly we study here is connected with the Weyl symmetry of the action of a classical theory, defined on a curved spacetime with the metric $g_{\mu \nu}$, by the transformations

$$
g_{\mu v}(x) \rightarrow e^{\omega(x)} g_{\mu v}(x) \quad, \quad \psi_{j}(x) \rightarrow e^{\lambda_{j} \omega(x)} \psi_{j}(x)
$$

where $\psi_{j}$ are fields in the theory, and $\omega$ is an arbitrary function. The consequence of this symmetry is tracelessness of the energy-momentum tensor in the classical theory

$$
\delta_{\omega} S_{m}[\psi, g]=0 \quad \Longrightarrow \quad T_{\mu}^{\mu}(x)=0
$$

However, it was shown [1] that even in simplest QFT's (of free spin-0 and spin-1/2 fields) there is an anomaly already at the 1-loop level ${ }^{1}$

$$
\left\langle\left\langle T_{\mu}^{\mu}\right\rangle\right\rangle=\mathcal{A}_{W} \neq 0
$$

For theories in $D=4$ the trace anomaly has the form

$$
\mathcal{A}_{W}=a E_{2}+c\left(W_{\mu \nu \rho \sigma}\right)^{2}
$$

where $E_{2}$ is the second Euler (or Gauss-Bonnet) topological density, while $W_{\mu \nu \rho \sigma}$ is the Weyl tensor. The coefficients $a$ and $c$ are model dependent. Let us mention that the original claim, published by Capper and Duff in 1975, produced a disbelief among experts at the beginning (see [2]). However, soon it was understood that the result is correct. Afterwards it was shown that the coefficients $a$ and $c$ are not generically 1-loop saturated in interacting theories.

The question of trace anomalies can be approached from the formal side of consistency conditions. It was shown that the most general expression for the trace anomaly in $D=4$ is $[3,4]$

$$
\mathcal{A}_{W}=a E_{2}+c\left(W_{\mu \nu \rho \sigma}\right)^{2}+e P_{4}
$$

where $P_{4}$ is the Pontryagin topological term

$$
P_{4}=\frac{1}{32 \pi^{2}} \varepsilon^{\mu \nu \rho \sigma} R_{\mu \nu}^{\alpha \beta} R_{\rho \sigma \alpha \beta}
$$

Comparing (5) with (4) we see that the consistency conditions allow the additional term in the trace anomaly, which we shall call the Pontryagin trace anomaly. It stands out in (5) by being parity-odd.

A glance at the literature on the trace anomaly reveals that the Pontryagin trace anomaly was mainly ignored by the community. ${ }^{2}$ The only explicit examples of interacting field theories which realise this anomaly are plagued by problems with unitarity [5]. To this one can add that the Pontryagin trace anomaly may not be compatible with SUSY [6].

\footnotetext{
${ }^{1}$ In our notation $\langle\langle\cdots\rangle\rangle$ denotes the time-ordered vacuum expectation value in a curved spacetime, while $\langle\cdots\rangle$ is the same in the flat spacetime.

${ }^{2}$ The Pontrygin trace anomaly was introduced in [4] and then unburied more then 25 yers later in [5].
} 


\section{Weyl fermions and Pontryagin trace anomaly}

\subsection{Original calculation}

In [7] parity-odd part of the trace anomaly for the free Weyl fermions in a curved four-dimensional spacetime was explicitly calculated for the first time. The action is given by

$$
S_{L}[g]=\frac{i}{2} \int d^{4} x \sqrt{|g|} \overline{\psi_{L}}\left(\gamma^{\mu} D_{\mu}-\overleftarrow{D}_{\mu} \gamma^{\mu}\right) \psi_{L}
$$

where, for the clarity of presentation, we assumed that the Weyl fermion is left-handed, $\gamma_{5} \psi_{L}=\psi_{L}$, and $D_{\mu}=\partial_{\mu}+\omega_{\mu}\left(\overleftarrow{D}_{\mu}=\overleftarrow{\partial}_{\mu}-\omega_{\mu}\right)$ acts only on the spinor $\psi_{L}\left(\overline{\psi_{L}}\right)$. The energy momentum tensor

$$
T^{\mu v}=\frac{i}{4} \overline{\psi_{L}}\left(\gamma^{\mu} D^{v}-\overleftarrow{D}^{v} \gamma^{\mu}\right) \psi_{L}+(\mu \leftrightarrow v)
$$

is both (covariantly) conserved and traceless on-shell, at least classically.

The calculation is done perturbatively around the Minkowski space, as a power expansion in the background field $h_{\mu \nu} \equiv g_{\mu \nu}-\eta_{\mu \nu}$. As the lowest order terms in $\left\langle\left\langle T_{\mu}^{\mu}\right\rangle\right\rangle$, are, by Eq. (5), expected to be of the order $h^{2}$, to calculate them we need to expand the action up to $h^{2}$ order and calculate corresponding vertices. Now, the calculation drastically simplifies if one makes the field redefinition $\psi_{L} \rightarrow|g|^{1 / 4} \psi_{L}$, and this was used in [7]. In this way one gets the following vertices up to $h^{2}$ order

$$
\begin{array}{rlrl}
V_{f f h} & : & & -\frac{i}{8}\left[\left(p+p^{\prime}\right)_{\mu} \gamma_{v}+\left(p+p^{\prime}\right)_{v} \gamma_{\mu}\right] \frac{1+\gamma_{5}}{2} \\
V_{f f h h} & : & \frac{1}{64} t_{\mu \nu \mu^{\prime} v^{\prime} \kappa \lambda}\left(k-k^{\prime}\right)^{\lambda} \gamma^{\kappa} \frac{1+\gamma_{5}}{2}
\end{array}
$$

where

$$
t_{\mu \nu \mu^{\prime} v^{\prime} \kappa \lambda}=\eta_{\mu \mu^{\prime}} \epsilon_{\nu v^{\prime} \kappa \lambda}+\eta_{\nu v^{\prime}} \epsilon_{\mu \mu^{\prime} \kappa \lambda}+\eta_{\mu v^{\prime}} \epsilon_{\nu \mu^{\prime} \kappa \lambda}+\eta_{\nu \mu^{\prime}} \epsilon_{\mu v^{\prime} \kappa \lambda}
$$

Using dimensional regularisation it was shown in [7] that the parity-odd part of the vacuum expectation value of the trace of the energy momentum tensor at $h^{2}$ order is given by

$$
\left\langle\left\langle T_{\mu}^{\mu}(x)\right\rangle\right\rangle_{\mathrm{P}-\mathrm{odd}}=\frac{1}{2} \int d^{4} x_{1} \int d^{4} x_{2} \mathcal{T}^{\mu_{1} v_{1} \mu_{2} v_{2}}\left(x, x_{1}, x_{2}\right) h_{\mu_{1} v_{1}}\left(x_{1}\right) h_{\mu_{2} v_{2}}\left(x_{2}\right)+O\left(h^{3}\right)
$$

where

$$
\mathcal{T}^{\mu_{1} v_{1} \mu_{2} v_{2}}\left(x, x_{1}, x_{2}\right)=-\eta_{\mu \nu}\left\langle T_{(0)}^{\mu v}(x) T_{(0)}^{\mu_{1} v_{1}}\left(x_{1}\right) T_{(0)}^{\mu_{2} v_{2}}\left(x_{2}\right)\right\rangle_{\mathrm{P}-\text { odd }}
$$

$T_{(0)}^{\mu \nu}$ is the energy momentum tensor in the flat space

$$
\left.T_{(0)}^{\mu v} \equiv 2 \frac{\delta S^{\prime}}{\delta h_{\mu v}(x)}\right|_{h=0}=-\frac{i}{4}\left(\overline{\psi_{L}} \gamma^{\mu} \stackrel{\leftrightarrow}{\partial^{v}} \psi_{L}+(\mu \leftrightarrow v)\right)
$$

where $S^{\prime}$ is the action after the field redefinition $\psi_{L} \rightarrow|g|^{1 / 4} \psi_{L}$ was performed. At the order $h^{2}$ one can check only the vertex $V_{f f h}$ is needed because the bubble diagram involving $V_{f f h h}$ gives a vanishing parity-odd contribution. The explicit calculation with dimensional regularization gives [7]

$$
\left\langle\left\langle T_{\mu}^{\mu}\right\rangle\right\rangle_{\mathrm{P}-\text { odd }}=\frac{i}{768 \pi^{2}} \epsilon^{\mu \nu \lambda \rho}\left(\partial_{\mu} \partial_{\sigma} h_{\nu}^{\tau} \partial_{\lambda} \partial_{\tau} h_{\rho}^{\sigma}-\partial_{\mu} \partial_{\sigma} h_{\nu}^{\tau} \partial_{\lambda} \partial^{\sigma} h_{\tau \rho}\right)+O\left(h^{3}\right)
$$

The quadratic term above is proportional to the quadratic part in the expansion of the Pontryagin density (6) in $h_{\mu v}$, with the coefficient being equal to $i / 48$. Comparing with (5), the conclusion of [7] 
was that the left-handed Weyl fermion in 4D has a non-vanishing Pontryagin trace anomaly with the coefficient $e$ given by

$$
e_{L}=\frac{i}{48}
$$

For the right-handed Weyl fermion one obtains a similar result with the coefficient $e_{R}=-e_{L}$. Note that the result implies that there is no Pontryagin trace anomaly for Majorana or Dirac fermions.

In the follow-up paper [8] it was shown that the result is compatible with diff-covariance, more precisely that the parity-odd counterterms one needs to add to the action to make $T_{\mu \nu}$ conserved are Weyl-invariant, and so do not contribute to the trace anomaly.

There are several surprising aspects of this result. In $D=4$ there is a (classical) one-to-one mapping between the Weyl and the massless Majorana fermion in curved space, and Majorana fermion does not exhibit Pontyagin trace anomaly. Note also that the coefficient in Pontryagin trace anomaly is imaginary number. That is the reason why the results from $[7,8]$ raised at the beginning some skepticism. We shall return to comment on these issues in the concluding section, after showing that this result survives some additional checks.

\subsection{Improved calculation}

Though the calculations presented in [7, 8] were performed in the standard way, the controversy which surrounds the result requests that calculation should be purified from all potential sources of error. This is the reason why in [11] we reconsidered the calculation without using simplifying field redefinition $\psi_{L}^{\prime}=|g|^{1 / 4} \psi_{L}$, and taking into account contributions coming also from 1-point and 2-point functions.

In this case one has additional vertices, aside those in (9)-(10). The relevant ones, up to $h^{2}$ order, are

$$
\begin{aligned}
& \left.V_{f f h}^{\prime}: \quad \frac{i}{4} \eta_{\mu v}(\not p+\not p)^{\prime}\right) P_{L} \\
& V_{f f h h}^{\prime}: \quad \frac{3 i}{64}\left[\left(\left(p+p^{\prime}\right)_{\mu} \gamma_{\mu^{\prime}} \eta_{\nu v^{\prime}}+\left(p+p^{\prime}\right)_{\mu} \gamma_{\nu^{\prime}} \eta_{\nu \mu^{\prime}}+\{\mu \leftrightarrow v\}\right)\right. \\
& \left.+\left(\left(p+p^{\prime}\right)_{\mu^{\prime}} \gamma_{\mu} \eta_{v v^{\prime}}+\left(p+p^{\prime}\right)_{\mu^{\prime}} \gamma_{v} \eta_{\mu v^{\prime}}+\left\{\mu^{\prime} \leftrightarrow v^{\prime}\right\}\right)\right] P_{L} \\
& V_{f f h h}^{\prime \prime}:-\frac{i}{16}\left[\eta_{\mu \nu}\left(\left(p+p^{\prime}\right)_{\mu^{\prime}} \gamma_{\nu^{\prime}}+\left(p+p^{\prime}\right)_{\nu^{\prime}} \gamma_{\mu^{\prime}}\right)+\eta_{\mu^{\prime} \nu^{\prime}}\left(\left(p+p^{\prime}\right)_{\mu} \gamma_{\nu}+\left(p+p^{\prime}\right)_{v} \gamma_{\mu}\right)\right] P_{L} \\
& V_{f f h h}^{\prime \prime \prime}: \quad \frac{i}{8}\left(\not p+\not p^{\prime}\right)\left(\eta_{\mu \nu} \eta_{\mu^{\prime} v^{\prime}}-\eta_{\mu v^{\prime}} \eta_{\mu^{\prime} v}-\eta_{\mu \mu^{\prime}} \eta_{v v^{\prime}}\right) P_{L}
\end{aligned}
$$

We have shown that (12)-(13) still applies, however the flat-space energy momentum is now

$$
\left.T_{(0)}^{\mu \nu} \equiv 2 \frac{\delta S}{\delta h_{\mu \nu}(x)}\right|_{h=0}=-\frac{i}{4}\left(\overline{\psi_{L}} \gamma^{\mu} \stackrel{\leftrightarrow}{\partial^{v}} \psi_{L}+(\mu \leftrightarrow v)\right)+\frac{i}{2} \eta_{\mu \nu} \overline{\psi_{L}} \gamma^{m} \stackrel{\leftrightarrow}{\partial_{m}} \psi_{L}
$$

which differs from (14) by the rightmost term. ${ }^{3}$ When (21) is used in (13), we see that now we have two vertices which are relevant, $V_{f f h}$ which as before represents the first term in (21), and $V_{f f h}^{\prime}$ which represents the second term.

The only nonvanishing contributions in the triangle diagram appear when either all three vertices are $V_{f f h}$, or when the first vertex $(x)$ is $V_{f f h}^{\prime}$ and the remaining two vertices $\left(x_{1}\right.$ and $\left.x_{2}\right)$ are $V_{f f h}$. The

\footnotetext{
${ }^{3}$ Obviously, (21) is on-shell the same as (14). However, we want to practice here extra caution, so we keep the full off-shell expression.
} 
first option of course reproduces the contribution to the P-odd trace anomaly from the previous section, which is (15), while the second option gives the contribution which is (-4) times (15). All together, a naive interpretation suggests that we obtain the Pontryagin trace anomaly with the coefficient $e_{L}=$ $-i / 16$, which is (-3) times the result found in [7] and stated in (16).

\subsubsection{Conservation of the energy-momentum tensor}

Before interpreting the above obtained results as a true P-odd trace anomaly, we have to check the diffcovariance. Due to the fact that there are no diff-anomalies in $4 \mathrm{D}$, properly defined energy momentum tensor must satisfy

$$
\nabla_{\mu}\left\langle\left\langle T^{\mu v}\right\rangle\right\rangle=0
$$

We have shown that for the left-handed Weyl fermion the P-odd part of the right hand side is

$$
\nabla_{\mu}\left\langle\left\langle T^{\mu v}\right\rangle\right\rangle_{\mathrm{P}-\text { odd }}=\frac{1}{2} \int d^{4} x_{1} \int d^{4} x_{2} h_{\mu_{1} v_{1}}\left(x_{1}\right) h_{\mu_{2} v_{2}}\left(x_{2}\right) \partial_{\mu}^{x}\left\langle T_{(0)}^{\mu v}(x) T_{(0)}^{\mu_{1} v_{1}}\left(x_{1}\right) T_{(0)}^{\mu_{2} v_{2}}\left(x_{2}\right)\right\rangle_{\mathrm{P}-\text { odd }}+O\left(h^{3}\right)
$$

where $T_{(0)}^{\mu v}$ is as in (21). Using dimensional regularisation, we have found that the $h^{2}$ term does not vanish. To fulfill (22), this means that one has to add a particular counterterm to the classical action which cancels this contribution. We have found out in our case that the part of the counterterm which is not invariant under infinitesimal Weyl transformations is given by

$$
C=-\frac{1}{2} \int d^{4} x h_{\mu}^{\mu} \mathcal{A}_{2}
$$

where $\mathcal{A}_{2}$ is the $h^{2}$ part of the P-odd trace anomaly (15). Now, this counterterm produces the contribution to the trace anomaly which is exactly $(-4)$ times the $h^{2}$ part of (15). After adding this contribution to the 1-loop contribution calculated above, we obtain that the result is consistent with the Pontryagin trace anomaly with the coefficient given by

$$
e_{L}=-\frac{i}{16}+\frac{i}{12}=\frac{i}{48}
$$

This is the same result as in (15)-(16), which confirms the claim from [7].

\subsection{Chirally coupled gravity}

While the improved calculation reduces the possibility of technical errors in [7], one would also like to have a different derivation, possibly done with different regularisation schemes. An important step in this direction would be to find a way to work with the Dirac instead of the Weyl fermion. An inspiration can be found in the Bardeen's method to chiral gauge anomalies in gauge theories $[9,10]$. It consists in introducing both a vector and axial potential as external sources of a Dirac fermion, and the usual consistent and covariant anomalies are obtained as specific limits.

Following this idea, in [11] we introduced a Metric-Axial-Tensor (MAT) Gravity, in which there is an axial partner of $g_{\mu \nu}$, which we denote $f_{\mu \nu}$. The MAT metric is then defined as

$$
G_{\mu \nu}=g_{\mu \nu}+\gamma_{5} f_{\mu \nu}
$$

which is then used to define all other MAT geometrical objects, including connection, vielbein $E_{a}^{\mu}$, spin connection $\Omega_{\mu}$ and Riemann tensor $\mathbf{R}_{\mu \nu \rho \sigma}$. The details of the construction are spelled out in [11]. 
Here we are interested in coupling Dirac fermion $\psi$ to such MAT background. ${ }^{4}$ This is achieved by taking the action of the theory to be

$$
S_{D}[G]=i \int d^{4} x \bar{\psi} \sqrt{|\bar{G}|} \gamma^{a} E_{a}^{\mu}\left(\partial_{\mu}+\frac{1}{2} \Omega_{\mu}\right) \psi
$$

The action is invariant on MAT diffeomorphisms with gauge parameter $\Theta=\xi+\gamma_{5} \zeta$, leading to two independent energy-momentum tensors which are classically covariantly conserved. If we write the action in the form $S=\int d^{4} x \bar{\psi} \sqrt{|\bar{G}|} O \psi$, the two conserved tensors can be written as

$$
T^{\mu \nu}=2 \bar{\psi} \frac{\delta O}{\delta G_{\mu \nu}} \psi \quad, \quad T_{5}^{\mu \nu}=2 \bar{\psi} \frac{\delta O}{\delta G_{\mu \nu}} \gamma_{5} \psi
$$

which refer to invariance on (standard) $\xi$-diffeomorphisms and (chiral) $\zeta$-diffeomorphism. In the zeroth order in expansion in $H_{\mu \nu} \equiv G_{\mu \nu}-\eta_{\mu \nu}=h_{\mu \nu}+\gamma_{5} f_{\mu \nu}$ (expansion around the flat space), (27) are given by

$$
T_{(0)}^{\mu \nu}=-\frac{i}{4}\left(\bar{\psi} \gamma^{\mu} \stackrel{\leftrightarrow}{\partial^{v}} \psi+\mu \leftrightarrow \nu\right) \quad, \quad T_{5(0)}^{\mu \nu}=\frac{i}{4}\left(\bar{\psi} \gamma_{5} \gamma^{\mu} \stackrel{\partial}{\nu}^{v} \psi+\mu \leftrightarrow \nu\right)
$$

The action (26) is also invariant under MAT Weyl transformtions of the metric, which produce the following two trace conditions in the classical theory

$$
\mathcal{T} \equiv T^{\mu v} g_{\mu v}+T_{5}^{\mu v} f_{\mu \nu}=0 \quad, \quad \mathcal{T}_{5} \equiv T^{\mu v} f_{\mu v}+T_{5}^{\mu v} g_{\mu \nu}=0
$$

the first one connected to the standard, and the second one to the chiral Weyl rescaling.

In [11] we have shown, by using dimensional regularisation, that the trace conditions (29) are anomalous. Again, we performed calculation up to the $H^{2}$ order (the lowest nonvanishing), to which only 3-point correlators of (28) contribute. A P-odd trace anomaly contribution comes from correlators containing odd number of $T_{5(0)}^{\mu \nu}$ operators, which results in the following expressions

$$
\begin{aligned}
& \left\langle\left\langle\mathcal{T}^{\mu v} \eta_{\mu \nu}\right\rangle\right\rangle=-\frac{i}{384 \pi^{2}} \epsilon^{\mu \nu \lambda \rho}\left(\partial_{\mu} \partial_{\sigma} h_{\nu}^{\tau} \partial_{\lambda} \partial_{\tau} f_{\rho}^{\sigma}-\partial_{\mu} \partial_{\sigma} h_{\nu}^{\tau} \partial_{\lambda} \partial^{\sigma} f_{\tau \rho}\right)+O\left(H^{3}\right) \\
& \left\langle\left\langle\mathcal{T}_{5}^{\mu \nu} \eta_{\mu \nu}\right\rangle\right\rangle=-\frac{i}{768 \pi^{2}} \epsilon^{\mu \nu \lambda \rho}\left(\partial_{\mu} \partial_{\sigma} h_{\nu}^{\tau} \partial_{\lambda} \partial_{\tau} h_{\rho}^{\sigma}-\partial_{\mu} \partial_{\sigma} h_{\nu}^{\tau} \partial_{\lambda} \partial^{\sigma} h_{\tau \rho}\right)+(h \rightarrow f)+O\left(H^{3}\right)
\end{aligned}
$$

which give the leading contributions to $\langle\langle\mathcal{T}\rangle\rangle$ and $\left\langle\left\langle\mathcal{T}_{5}\right\rangle\right\rangle$.

For the purposes of interpreting these results it is convenient to pass to the chiral basis. This is achieved by the metric (25) in the following way

$$
G_{\mu \nu}=P_{L} g_{\mu \nu}^{(L)}+P_{R} g_{\mu \nu}^{(R)} \quad, \quad g_{\mu \nu}^{(L, R)}=g_{\mu \nu} \pm f_{\mu \nu}
$$

All the MAT objects we mentioned follow naturally chiral separation. Vierbein, connection and Riemann tensor satisfy $X[G]=P_{L} X\left[g_{(L)}\right]+P_{R} X\left[g_{(R)}\right]$, while the action (26) satisfies

$$
S_{D}[G]=S_{L}\left[g_{L}\right]+S_{R}\left[g_{R}\right]
$$

i.e., the theory describes massless Dirac field whose left and right components are coupled to metrics $g_{\mu \nu}^{(L)}$ and $g_{\mu \nu}^{(R)}$, respectively. ${ }^{5}$

\footnotetext{
${ }^{4}$ As $\gamma_{5}^{2}=1$, this construction can be viewed as a representation of pseudo-complex numbers.

${ }^{5}$ In this sense, MAT gravity is an example of a bimetric theory.
} 


\subsubsection{The Pontryagin anomaly for the Weyl fermion}

The form of the action (33) shows that if we choose $g_{\mu \nu}^{(R)}=\eta_{\mu \nu}$, which means $f_{\mu \nu}=h_{\mu \nu}$, then we have a theory in which the right handed component of the Dirac field is completely free and so cannot contribute to anomalies. The anomalies can come only from the left handed Weyl component, which is coupled to the curved spacetime with the metric $g_{\mu \nu}^{(L)}=\eta_{\mu \nu}+2 h_{\mu \nu}$. Using this in the (30) we see that our result for the P-odd part of the standard trace anomaly is, up to order $h^{2}$, consistent with the expression

$$
\left\langle\left\langle\mathcal{T}_{L}\right\rangle\right\rangle=\left\langle\left\langle\left(\mathcal{T}+\mathcal{T}_{5}\right)\right\rangle\right\rangle=\frac{i}{48} P_{4}\left[g_{L}\right]
$$

which is nothing but the Pontryagin trace anomaly with coefficient (16). Remembering now that this is the anomaly connected with the left-handed Weyl fermion coupled to the metric $g_{\mu \nu}^{(L)}$, we reconfirm the result of [7]. As a check, we can calculate anomaly contribution of the right-handed component

$$
\left\langle\left\langle\mathcal{T}_{R}\right\rangle\right\rangle=\left\langle\left\langle\left(\mathcal{T}-\mathcal{T}_{5}\right)\right\rangle\right\rangle=0
$$

which is as expected because in this choice of background the right-handed component is coupled to the flat metric.

If we instead took $g_{\mu \nu}^{(L)}=\eta_{\mu \nu}$, procedure repeats with the exchange $L \leftrightarrow R$. The only difference is that now we have $f_{\mu \nu}=-h_{\mu \nu}$, which produces a minus sign in (34), so we obtain Pontryagin trace anomaly for right handed Weyl fermion with the coefficient $e_{R}=-e_{L}=-\frac{i}{48}$.

\subsubsection{The Pontryagin anomaly for Dirac and Majorana fermions}

It is obvious that if we choose $f_{\mu \nu}=0$ our theory describes the massless Dirac fermion minimally coupled to the metric $g_{\mu \nu}$. In this case the P-odd part of the trace anomaly is given by (30) which obviously vanishes at the lowest order in the expansion around the flat space. As the Majorana fermion is the Dirac fermion with imposed reality condition, the same applies to it. Again, this is in accord with [7].

\section{Discussion and outlook}

By presenting multiple calculations we hope that we have established a strong evidence that QFT with free Weyl fermions in a generic curved four-dimensional spacetime displays parity-odd trace anomaly of the Pontryagin type, when regulated with dimensional regularisation. Though Pontryagin trace anomaly is allowed from the purely cohomological analysis, this is unexpected result which was (and still mostly is) received with a disbelief.

Let us briefly consider the main opposing argument. It is based on the fact that actions for the free Weyl fermion and the free massless Majorana fermion, defined on a curved spacetime, are classically equivalent. It is a part of a widespread wisdom to take as granted that the quantum theories are also equivalent. In the last step, one notices that the Majorana fermion cannot produce the Pontryagin trace anomaly, and so, the claim goes, neither can the Weyl fermion. However, one must notice that assumption that a classical property implies a quantum property is far from trivial, in fact anomalies in general are exactly examples in which this logic is violated. Note that the massless Majorana fermion and the Weyl fermion in 4D belong to two different Lorentz representations, irreducible to each other. So, one cannot a priori exclude the possibility that massless Majorana and Weyl fermions, when defined on curved space, do not have the same path integral measure and are different when quantised. Let us add here that the claim apparently opposite to our, made in [12], also rests on the 
assumption of massless Majorana / Weyl equivalence, and so just proves that the massless Majorana fermion does not produce the Pontryagin trace anomaly, an uncontroversial claim supported also by our calculations. For the detailed account of (and respond to) criticism, and we direct readers to Refs. $[7,8,11]$.

We believe that in instances like this it is wise to accept "shut up and calculate" approach, which is what we did. After the original result was confirmed and then reconfirmed, and that in every new calculation new people were involved, possibilities of a calculational/technical errors are drastically reduced. In view of this, let us now accept the result and analyse possible consequences.

As all calculations reviewed here use the dimensional regularisation, it may be that the regularisation breaks down in this particular case. While we cannot dismiss this scenario, we are strongly doubting it. The calculation is similar to the one for Kimura-Delbourgo-Salam anomaly [13, 14], which is undisputed. Also, the dimensional regularisation is considered to be the most powerful and economic regularisation technique, with widespread use (and included in almost all modern textbooks on QFT). A failure of the dimensional regularisation would have profound consequences, so it is important to check the result by using different regularisations.

Let us now assume that the Pontryagin trace anomaly of the Weyl fermion survives additional checks, and is taken as granted. Observe that it has the imaginary coefficient, which is rather strange and could be a sign of a breakdown of unitarity. This would be in line with [5], where it was found in some explicit examples of interacting theories that the presence of the Pontryagin trace anomaly is accompanied by unitarity issues. If this Pontryagin trace anomaly is indeed harmful, the result becomes profound, and with serious consequences. E.g., it would mean that the original "minimal" Standard Model with neutrinos described as Weyl fermions of only one chirality is not well-defined theory in generic curved spacetime. To avoid the harmful anomaly, they must be either Majorana, or come into Weyl pairs of both chirality (which means they are Dirac), with the mass matrix then being natural addition (as seen in Nature). If this Pontryagin trace anomaly is harmless, it is still important as it introduces a mechanism for gravitational breaking of CP symmetry, and contributes to the gravitational Schwinger effect. Some of its phenomenological consequences are analysed in [15].

As for the outlook, obviously the first task is to do the calculation with different regularisations. Such confirmation is necessary to fully trust the result. If the anomaly survives further independent checks, we need to understand the imaginary nature of anomaly and see whether there are problems with unitarity and whether the anomaly is harmless or harmful. Some of these calculations are already under way.

\section{Acknowledgements}

This research has been supported by the Croatian Science Foundation under the project No. 8946 and by the University of Rijeka under the research support No. 13.12.1.4.05. Finally, A.D.P. acknowledges funding by the DFG, Grant Ei/1037-1.

\section{References}

[1] D. M. Capper and M. J. Duff, Nuovo Cim. A 23, 173 (1974); Phys. Lett. A 53, 361 (1975).

[2] M. J. Duff, Class. Quant. Grav. 11, 1387 (1994).

[3] L. Bonora, P. Pasti, M. Tonin, Phys. Lett. 149B, 346 (1985).

[4] L. Bonora, M. Bregola, P. Pasti, Class. Quant. Grav. 3, 635 (1986).

[5] Y. Nakayama, Nucl. Phys. B 859, 288 (2012); Int. J. Mod. Phys. Conf. Ser. 21, 63 (2013).

[6] L. Bonora and S. Giaccari, JHEP 1308, 116 (2013). 
[7] L. Bonora, S. Giaccari and B. Lima de Souza, JHEP 1407, 117 (2014)

[8] L. Bonora, A. D. Pereira and B. L. de Souza, JHEP 1506, 024 (2015)

[9] W. A. Bardeen, Phys. Rev. 184, 1848 (1969).

[10] A. Andrianov, L. Bonora, Nucl. Phys. B233, 232 (1984); Nucl. Phys. B233, 247 (1984).

[11] L. Bonora, M. Cvitan, P. Dominis Prester, A. Duarte Pereira, S. Giaccari and T. Štemberga, Eur. Phys. J. C 77, 511 (2017).

[12] F. Bastianelli and R. Martelli, JHEP 1611, 178 (2016).

[13] T. Kimura, Prog. Theor. Phys. 42, 1191 (1969).

[14] R. Delbourgo and A. Salam Phys. Lett. 40B, 381 (1972).

[15] S. Mauro and I. L. Shapiro, Phys. Lett. B 746, 372 (2015). 